\title{
Numerical Integration of Average Run Length of CUSUM Control Chart for ARMA Process
}

\author{
S. Phanyaem, Y. Areepong, and S. Sukparungsee
}

\begin{abstract}
The main purpose of this paper is to present the numerical integration of average run length (ARL) for the Cumulative Sum (CUSUM) control chart. In addition, we compare the ARL between the cumulative sum (CUSUM) and the exponentially weighted moving average (EWMA) control charts for an autoregressive and moving average process, $\operatorname{ARMA}(p$, q) with exponential distribution white noise. The results show that the EWMA control chart is superior to the CUSUM control chart when the process has small shifts in the process mean.
\end{abstract}

Index Terms-Autoregressive and moving average (ARMA), cumulative sum (CUSUM), exponentially weighted moving average (EWMA), average run length (ARL).

\section{INTRODUCTION}

Statistical Process Control (SPC) charts are widely used in many areas of applications such as economic, finance, medicine and engineering. It used in monitoring, measuring, controlling and improving quality in areas such as industrial statistics and manufacturing [1]. The control charts are specialized time series plots which assist in determining whether a process is in statistical control. Some of the most widely used forms of control charts are X-R control chart and Individual control charts. There are frequently referred to as "Shewhart" control chart after the control charting pioneer, Walter Shewhart, who introduced such techniques. These control charts are sensitive to detecting relatively large shifts in the process $(\geq 1.5 \sigma)$. Two types of control charts are used to detect smaller shifts $(<1.5 \sigma)$ namely Cumulative Sum (CUSUM) control chart and Exponentially Weighted Moving Average (EWMA) control charts. The CUSUM control chart has been proposed as good alternatives to the Shewhart control chart for detecting small changes in process means. It was first proposed by Page [2].

The EWMA control chart was initially proposed by Robert [3]. It is usually used to monitor and detect a small change in a process mean. The EWMA control chart is based on a weighted average of current and previous data.

There are many situations in which the process is autocorrelated such as in chemical process, so it needs to be monitored by appropriate control charts. The varieties of CUSUM and EWMA control charts have been developed [4]-[8]. However, the studies of control charts in detecting the mean changes in autocorrelated process in the above papers are mainly based on numerical simulations of ARL.

Manuscript received May 15, 2014; revised July 15, 2014.

The authors are with the Faculty of Applied Science, King Mongkut's University of Technology North Bangkok, Bangkok, 10800, Thailand (e-mail: suvimolp@kmutnb.ac.th, yupaporna@kmutnb.ac.th, swns@kmutnb.ac.th; tel.: 662-555-2000; fax: 662-585-6105).
The ARL is a traditional measurement of control chart's performance, the expected number of observations taken from an in-control process until the control chart falsely signals out-of-control is denoted by $\mathrm{ARL}_{0}$. $\mathrm{An} \mathrm{ARL}_{0}$ will be regarded as acceptable if it is large enough to keep the level of false alarms at an acceptable level. A second common characteristic is the expected number of observations taken from an out-of-control process until the control chart signals that the process is out-of-control is denoted by $\mathrm{ARL}_{1}$. There are several methods that can be utilized to find the ARL such as Markov Chain approach (MCA), Integral Equation approach (IE) and Monte Carlo simulations (MC).

Sukparungsee [9] have used the Martingale approach to derive the analytical formulas of the Average Run Length (ARL) and the Average Delay (AD) in the case of Gaussian and some Non-Gaussian distributions. Later, Areepong [10] derived the explicit formulas of ARL and AD for EWMA control chart in the case of Exponential distribution. Recently, Busaba [11] was analyzed the explicit formulas of ARL for CUSUM control chart, its corresponding in the case of a Stationary First Order Autoregressive, AR(1) process with exponential white noise.

The objective of this paper is to propose the numerical integration of $\mathrm{ARL}$ for CUSUM control chart for $\operatorname{ARMA}(p, q)$ process with exponential white noise and compare it with the EWMA control chart. The organization of this paper is as follows: In Section II, the characteristic of CUSUM control chart for $\operatorname{ARMA}(p, q)$ is presented. The numerical integration of ARL for CUSUM control chart is proposed in Section III. The numerical method of ARL for EWMA control chart is discussed in Section IV. The comparison of performance of the ARL between CUSUM and EWMA charts are presented in Section V. Conclusions are provided in the final section.

\section{THE CUSUM CONTROL CHART FOR ARMA $(P, Q)$}

In this section we describe the characteristics of the CUSUM control chart, its was originally introduced by Page [2] in quality control in order to detect a small shift in the mean of a process as soon as it occurs.

Let $C_{t}$ be the CUSUM statistics, the recursive CUSUM based on $\operatorname{ARMA}(p, q)$ process is defined as:

$$
C_{t}=\max \left(C_{t-1}+X_{t}-a, 0\right) ; t=1,2, \ldots
$$

where $X_{t}$ is a sequence of $\operatorname{ARMA}(p, q)$ process, $C_{0}=u$ is an initial value, $a$ is a reference value of CUSUM chart.

The $\operatorname{ARMA}(p, q)$ process described by the following recursion 


$$
X_{t}=\mu+\varphi_{1} X_{t-1}+\ldots+\varphi_{p} X_{t-p}+\xi_{t}-\theta_{1} \xi_{t-1}-\ldots-\theta_{q} \xi_{t-q}
$$

where $\xi_{t}$ is a white noise process assumed with exponential distribution, $\varphi_{i}$ is an autoregressive coefficient, $i=1,2, \ldots, p$;

$0 \leq \varphi_{i} \leq 1$ and $\theta_{i}$ is moving average coefficient, $i=1,2, \ldots, q$;

$0 \leq \theta_{i} \leq 1$. Assume the initial value of $\operatorname{ARMA}(p, q)$ process $\xi_{t-1}, \xi_{t-2}, \ldots, \xi_{t-p}$ and $X_{t-1}, X_{t-2}, \ldots, X_{t-p}=1$.

The stopping time of CUSUM control chart is given by

$$
\tau_{h}=\inf \left\{t>0 ; C_{t}>h\right\}, h>u
$$

where $\tau_{h}$ is the stopping time

$h$ is the upper control limit (UCL).

Let $H(u)$ denote the ARL for $\operatorname{ARMA}(p, q)$ process with an initial value $C_{0}=u$. To define function $H(u)$ as follows

$$
A R L=H(u)=\mathbb{E}_{\infty}\left(\tau_{h}\right) .
$$

where $\mathbb{E}_{\infty}$ (.) is the expectation.

\section{NUMERICAL INTEGRATION OF ARL FOR CUSUM CHART}

In this section we propose the numerical scheme for solving the integral equation [12]. By quadrature rule approach we can approximate the integral by finite sum of areas of rectangles with base $h / m$ with heights chosen as the values of $f$ at midpoints of intervals of length $h / \mathrm{m}$ beginning at zero. Particularly, once the choice of a quadrature rule is made, the interval $[0, h]$ is divided into a partition $0 \leq a_{1} \leq a_{2} \leq \ldots \leq a_{m} \leq h$ and a set of constant weights $w_{j}=h / m \geq 0$.

The approximation for an integral equation as follows

$$
\int_{0}^{h} W(y) F(y) d y \approx \sum_{j=1}^{m} w_{j} F\left(a_{j}\right)
$$

where $W(y)$ and $F(y)$ are given functions, $a_{j}$ is a set of point and $w_{j}$ is a weight define different quadrature rules.

In this section we present the numerical integration of ARL for CUSUM chart. The notations $\mathbf{P}_{c}$ denote the probability measure and $\mathbf{E}_{c}$ denote the expectation corresponding to an initial value $u$.

The solution of integral equation as follows

$$
H(u)=1+\mathbb{E}_{c}\left[I\left\{0<C_{1}<h\right\} H\left(C_{1}\right)\right]+\mathbb{P}_{c}\left\{C_{1}=0\right\} H(0) .
$$

Therefore, the integral equation of CUSUM control chart as follows:

$$
H(u)=1+\alpha e^{\alpha\left(u-a+\mu+\varphi_{1} X_{t-1}+\ldots+\varphi_{p} X_{t-p}-\theta_{1} \xi_{t-1}-\ldots-\theta_{q} \xi_{t-q}\right.} \int_{0}^{h} H(y) e^{-\alpha y} d y
$$

$$
+\left(1-e^{-\alpha\left(a-u-\mu-\varphi_{1} X_{t-1}-\ldots-\varphi_{p} X_{t-p}+\theta_{1} \xi_{t-1}+\ldots+\theta_{q} \xi_{t-q}\right)}\right) H(0)
$$

Firstly, the integral equation (7) can be rewritten as follows

$$
\begin{gathered}
H(u)=1+H(0) F\left(a-u-\mu-\varphi_{1} X_{t-1}-\ldots-\varphi_{p} X_{t-p}\right. \\
\left.+\theta_{1} \xi_{t-1}+\ldots+\theta_{q} \xi_{t-q}\right)+\int_{0}^{h} H(y) f(y+a-u-\mu \\
\left.-\varphi_{1} X_{t-1}-\ldots-\varphi_{p} X_{t-p}+\theta_{1} \xi_{t-1}+\ldots+\theta_{q} \xi_{t-q}\right) d y,
\end{gathered}
$$

where $F(u)=1-e^{-\lambda u}$ and $f(u)=\frac{d F(u)}{d u}=\lambda e^{-\lambda u}$.

Let $\tilde{H}(u)$ denote the approximated solution of $H(u)$ by using the quadrature rule, the integral equation (7) can be approximated by

$$
\begin{gathered}
\tilde{H}\left(a_{i}\right)=1+\tilde{H}\left(a_{1}\right) F\left(a-a_{i}-\mu-\varphi_{1} X_{t-1}-\ldots-\varphi_{p} X_{t-p}\right. \\
\left.+\theta_{1} \xi_{t-1}+\ldots+\theta_{q} \xi_{t-q}\right)+\sum_{j=1}^{m} w_{j} \tilde{H}\left(a_{j}\right) f\left(a_{j}+a-a_{i}\right. \\
\left.-\mu-\varphi_{1} X_{t-1}-\ldots-\varphi_{p} X_{t-p}+\theta_{1} \xi_{t-1}+\ldots+\theta_{q} \xi_{t-q}\right)
\end{gathered}
$$

Equation (9) is a system of $m$ linear equations in the $m$ unknowns $\tilde{H}\left(a_{1}\right), \tilde{H}\left(a_{2}\right), \ldots, \tilde{H}\left(a_{m}\right)$ can be re-arranged as

$$
\begin{gathered}
\tilde{H}\left(a_{1}\right)=1+\tilde{H}\left(a_{1}\right)\left[F \left(a-a_{1}-\mu-\varphi_{1} X_{t-1}-\ldots-\varphi_{p} X_{t-p}+\theta_{1} \xi_{t-1}\right.\right. \\
\left.+\ldots+\theta_{q} \xi_{t-q}\right)+w_{1} f\left(a-\mu-\varphi_{1} X_{t-1}-\ldots-\varphi_{p} X_{t-p}\right. \\
\left.+\theta_{1} \xi_{t-1}+\ldots+\theta_{q} \xi_{t-q}\right)+\sum_{j=2}^{m} w_{j} \tilde{H}\left(a_{j}\right) f\left(a_{j}+a-a_{1}\right. \\
\left.-\mu-\varphi_{1} X_{t-1}-\ldots-\varphi_{p} X_{t-p}+\theta_{1} \xi_{t-1}+\ldots+\theta_{q} \xi_{t-q}\right) \\
\tilde{H}\left(a_{2}\right)=1+\tilde{H}\left(a_{1}\right)\left[F \left(a-a_{2}-\mu-\varphi_{1} X_{t-1}-\ldots-\varphi_{p} X_{t-p}+\theta_{1} \xi_{t-1}\right.\right. \\
\left.+. .+\theta_{q} \xi_{t-q}\right)+w_{1} f\left(a_{1}+a-a_{2}-\mu-\varphi_{1} X_{t-1}-. .-\varphi_{p} X_{t-p}\right. \\
\left.\quad+\theta_{1} \xi_{t-1}+\ldots+\theta_{q} \xi_{t-q}\right)+\sum_{j=2}^{m} w_{j} \tilde{H}\left(a_{j}\right) f\left(a_{j}+a-a_{2}\right. \\
\left.\quad-\mu-\varphi_{1} X_{t-1}-\ldots-\varphi_{p} X_{t-p}+\theta_{1} \xi_{t-1}+\ldots+\theta_{q} \xi_{t-q}\right) \\
\quad \vdots \\
\tilde{H}\left(a_{m}\right)=1+\tilde{H}\left(a_{1}\right)\left[F \left(a-a_{m}-\mu-\varphi_{1} X_{t-1}-\ldots-\varphi_{p} X_{t-p}+\theta_{1} \xi_{t-1}\right.\right. \\
\left.+. .+\theta_{q} \xi_{t-q}\right)+w_{1} f\left(a_{1}+a-a_{m}-\mu-\varphi_{1} X_{t-1}-. .-\varphi_{p} X_{t-p}\right. \\
\left.\quad+\theta_{1} \xi_{t-1}+\ldots+\theta_{q} \xi_{t-q}\right)+\sum_{j=2}^{m} w_{j} \tilde{H}\left(a_{j}\right) f\left(a_{j}+a-a_{m}\right. \\
\left.-\mu-\varphi_{1} X_{t-1}-\ldots-\varphi_{p} X_{t-p}+\theta_{1} \xi_{t-1}+\ldots+\theta_{q} \xi_{t-q}\right)
\end{gathered}
$$

or in matrix form as follows:

$$
\mathbf{H}_{m \times 1}=\mathbf{1}_{m \times 1}+\mathbf{R}_{m \times m} \mathbf{H}_{m \times 1}
$$

where $\mathbf{H}_{m \times 1}=\left(\begin{array}{c}\tilde{H}\left(a_{1}\right) \\ \tilde{H}\left(a_{2}\right) \\ \vdots \\ \tilde{H}\left(a_{m}\right)\end{array}\right), \quad \mathbf{1}_{m \times 1}=\left(\begin{array}{c}1 \\ 1 \\ \vdots \\ 1\end{array}\right)$ 
and $\mathrm{I}_{m}=\operatorname{diag}(1,1, \ldots, 1)$ is the unit matrix of order $m$. If there exists $\left(\mathbf{I}_{m}-\mathbf{R}_{m \times m}\right)^{-1}$, then the solution of matrix equation as follows

$$
\mathbf{H}_{m \times 1}=\left(\mathbf{I}_{m}-\mathbf{R}_{m \times m}\right)^{-1} \mathbf{1}_{m \times 1} .
$$

Solving set of equations for the approximate values of $\tilde{H}\left(a_{1}\right), \tilde{H}\left(a_{2}\right), . ., \tilde{H}\left(a_{m}\right)$. Therefore, the numerical integration of ARL for CUSUM control chart as follows:

$$
\begin{gathered}
\tilde{H}(u)=1+\tilde{H}\left(a_{1}\right) F\left(a-u-\mu-\varphi_{1} X_{t-1}-\ldots-\varphi_{p} X_{t-p}\right. \\
\left.+\theta_{1} \xi_{t-1}+\ldots+\theta_{q} \xi_{t-q}\right)+\sum_{j=1}^{m} w_{j} \tilde{H}\left(a_{j}\right) f\left(a_{j}+a-u\right. \\
\left.-\mu-\varphi_{1} X_{t-1}-\ldots-\varphi_{p} X_{t-p}+\theta_{1} \xi_{t-1}+\ldots+\theta_{q} \xi_{t-q}\right)
\end{gathered}
$$

with $w_{j}=\frac{h}{m}$ and $a_{j}=\frac{h}{m}\left(j-\frac{1}{2}\right) ; j=1,2, \ldots, m$.

\section{NUMERICAL INTEGRATION OF ARL FOR EWMA CHART}

The EWMA control chart was proposed by Robert [3]. The recursive equation of EWMA statistics is defined by:

$$
Z_{t}=(1-\lambda) Z_{t-1}+\lambda X_{t} ; t=1,2, \ldots
$$

where $X_{t}$ is a sequence of $\operatorname{ARMA}(p, q)$ process, $Z_{0}=u$ is an initial value, $\lambda$ is an exponential smoothing parameter with $0<\lambda<1$.

Phanyaem [13] proposed the numerical method for solving integral equation of ARL for EWMA control chart when observations are $\operatorname{ARMA}(p, q)$. The numerical integration of ARL for EWMA control chart as follows

$$
\begin{gathered}
\tilde{L}(u) \approx 1+\frac{1}{\lambda} \sum_{j=1}^{m} w_{j} \tilde{L}\left(a_{j}\right) \\
\left.f\left(\frac{a_{j}-(1-\lambda) u}{\lambda}\right)-\mu-\varphi_{1} X_{t-1}-\ldots-\varphi_{p} X_{t-p}+\theta_{1} \xi_{t-1}+\ldots+\theta_{q} \xi_{t-q}\right)
\end{gathered}
$$

process between CUSUM and EWMA charts. The values of parameter for EWMA and CUSUM charts were established by setting $\mathrm{ARL}_{0}=370, \alpha_{0}=1, \varphi_{1}=0.10, \varphi_{2}=0.10, \theta_{1}=0.10$ and $\theta_{2}=0.10$.

TABLE I: COMPARISON OF ARL $\operatorname{AR}_{1}$ FOR ARMA $(1,1)$ BETWEEN CUSUM AND EWMA CONTROL CHARTS GIVEN $U=0$

\begin{tabular}{ccc}
\hline \hline Shift size & CUSUM chart & $\begin{array}{c}\text { EWMA chart } \\
\delta\end{array}$ \\
$a=2.5, h=3.665$ & $\lambda=0.20, b=0.222689$ \\
\hline 0.00 & 370.717 & 370.277 \\
0.01 & 347.618 & 63.707 \\
0.02 & 326.370 & 35.367 \\
0.03 & 306.797 & 24.718 \\
0.04 & 288.741 & 19.134 \\
0.05 & 272.064 & 15.696 \\
0.10 & 205.393 & 8.609 \\
0.20 & 125.734 & 4.948 \\
0.30 & 83.201 & 3.703 \\
0.40 & 58.567 & 3.072 \\
0.50 & 43.332 & 2.689 \\
\hline \hline
\end{tabular}

TABLE II: COMPARISON OF ARL $\operatorname{AR}_{1}$ FOR ARMA $(2,2)$ BETWEEN CUSUM AND EWMA CONTROL CHARTS GIVEN $U=0$

\begin{tabular}{ccc}
\hline \hline Shift size & CUSUM chart & EWMA chart \\
$\delta$ & $a=2.5, h=3.663$ & $\lambda=0.20, b=0.010033$ \\
\hline 0.00 & 370.052 & 370.018 \\
0.01 & 347.003 & 55.511 \\
0.02 & 325.800 & 30.538 \\
0.03 & 306.268 & 21.308 \\
0.04 & 288.250 & 16.501 \\
0.05 & 271.607 & 13.552 \\
0.10 & 205.069 & 7.499 \\
0.20 & 125.558 & 4.385 \\
0.30 & 83.0974 & 3.326 \\
0.40 & 58.5019 & 2.789 \\
0.50 & 43.2883 & 2.463 \\
\hline \hline
\end{tabular}

Comparing our results from the CUSUM and EWMA control charts shows that for the case of a one-sided shift, it has been shown that the EWMA control chart is the best control chart in the sense that it has minimizes the supremum of the conditional Average Run Length $\left(\mathrm{ARL}_{1}\right)$ when the process has a small shift $(\delta<0.50)$.

\section{CONCLUSION}

In this paper, we proposed the numerical integration of ARL for CUSUM control chart when observations are $\operatorname{ARMA}(p, q)$ with exponential white noise. In addition, we compare the efficientcy of control charts between CUSUM and EWMA control charts. The results show that the EWMA control chart is superior to the CUSUM control chart for all magnitude of shifts in the process mean.

\section{REFERENCES}

[1] B. Mason and J. Antony, "Statistical process control: an essential ingredient for improving service and manufacturing quality," Managing Service Quality, vol. 10, pp. 233-238, 2000.

[2] E. S. Page, "Continuous inspection schemes," Biometrika, vol. 41, 1954, pp. 100-114.

[3] S. W. Roberts, "Control Chart Test Based on Geometric Moving Average," Technometrics, vol. 1, pp. 239-250, 1959. 
[4] L. C. Alwan and H. V. Roberts, "Time-series modeling for statistical process control," Journal Business Econometric Statistics, vol. 6, pp. 87-95, 1998

[5] D. C. Montgomery and C. M. Mastrangelo, "Some statistical process control charts methods for autocorrelated data," Quality Technology, vol. 23, pp.179-193, 1991.

[6] E. Yashchin, "Performance of CUSUM control schemes for serially correlated observations," Technometrics, vol. 35, pp. 37-52, 1993.

[7] C. W. Lu and M. R. Reynolds, "EWMA Control charts for monitoring the mean of autocorrelated processes," Quality Technology, vol. 31, pp. 166-188, 1999.

[8] W. Jiang, K. L, Tsui, and W. H. Woodall, "A new SPC monitoring method: The ARMA chart," Technometrics, vol. 42, pp. 399-410, 2000.

[9] S. Sukparungsee and A. A. Novikov, "On EWMA procedure for detection of a change in observations via martingale approach," An International Journal of Science and Applied Science, vol. 6, pp. 373-380, 2006.

[10] Y. Areepong and A. A. Novikov, "An integral equation approach for analysis of control charts," Ph.D. dissertation, University of Technology, Australia, 2009.
[11] J. Busaba, S. Sukparungsee, Y. Areepong, and, G. Mititelu "Numerical approximations of average run length for $\mathrm{AR}(1)$ on exponential CUSUM," presented at the International Muti Conference of Engineers and Computer Scientists, Hong Kong, March 7-10, 2012.

[12] K. Atkinson and W. Han, Theoretical Numerical Analysis: A Functional Analysis Framework, New York: Springer Verlag, 2001.

[13] S. Phanyaem, Y. Areepong, S. Sukparungsee, and G. Mititelu, "Explicit formulas of average run length for $\operatorname{ARMA}(1,1)$," International Journal of Applied Mathematics and Statistics, vol. 43, 2013, pp. 392-405.

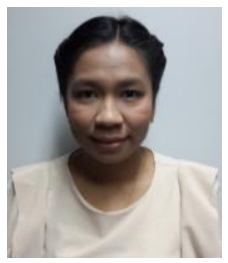

S. Phanyaem is currently a $\mathrm{Ph} . \mathrm{D}$. candidate in applied statistics, the Department of Applied Statistics, King Mongkut's University of Technology North Bangkok, Thailand. Her research interests include statistical process control and meta analysis. 